\title{
Analytical Review of Major Nocturnal Pests' Detection Technique using Computer Vision
}

\section{DEVEN J. PATEL ${ }^{1 *}$ and NIRAV BHATT ${ }^{2}$}

IInformation Technology Cell, Junagadh Agricultural University, Junagadh, Gujarat, India.

${ }^{2}$ Department of Computer Engineering, RK University, Kasturbadham, Rajkot, Gujarat, India.

\begin{abstract}
Research in agriculture is increasing quality and quantity, but pest reduces it. To prevent the effect of these pests, insecticides are used. But excessive use of pesticides is very harmful to production and environment. So initially pest detection is necessary. We work on nocturnal pests because that can be easily attracting using night trapping tools. The purpose of this review article is to analyse the popular techniques and find the right technique for the initial diagnosis and early detection of major nocturnal flying pests like Pink Bollworm, White Grub, Helicoverpa and Spodoptera. The importance of early detection can be in identifying and classifying the pests in a digital view. We have concluded our results with the various methods and the prospects of future research.
\end{abstract}

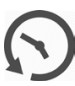

\section{Article History}

Received: 05 July 2018 Accepted:17 July 2018

\section{Keywords}

Computer Vision, Pest Detection Techniques, Convolution Neural

Networks,

Deep Learning.

\section{Introduction}

India has leading farm output worldwide. Cropping systems vary among farms depending on available resources and constraints. Farmers encounter many questions on these cropping systems depending on climate, diseases on crops, place, and type of land etc. ${ }^{1}$. The quality of agricultural products has decreased due to the presence of pests and diseases. Poverty, food insecurity and mortality will increase and the amount of food production will decrease because of the presence of pest on the crop is not adequately examined ${ }^{2}$. In general, methods to find plant pests are manual or use different trapping tools. One such main approach is bare eye inspection, but for this method need continuous monitoring of the farm by the person who has deep knowledge about the pest and its subsequent diseases ${ }^{3}$.

Gujarat is the main producer of cotton and groundnuts in India. Other major crops produced are cereal grains. My research is on major nocturnal flying insects of these crops. The pink bollworm (Pectinophora gossypiella) is being a pest in cotton farming. Groundnut crop is infested with sucking type of insects pests like white grub. Helicoverpa armigera is the serious pest for pepper crop. The fall armyworm Spodoptera is a prime pest for cereal

CONTACT Deven J. Patel djpatel@jau.in $\mathbf{9}$ Information Technology Cell, Junagadh Agricultural University, Junagadh, Gujarat,

India.

(c) (i)

(C) 2018 The Author(s). Published by Oriental Scientific Publishing Company.

This is an 6 Open Access article licensed under a Creative Commons license: Attribution 4.0 International (CC-BY).

Doi: http://dx.doi.org/10.13005/ojcst11.03.06 
crop. They are generally nocturnal and are usually seen flying after sunset. The use of light for sampling night-flying pest is a long-standing technique ${ }^{4}$. We are going to use the light trap to attracting pest for automatic detection for future research.

The computer vision is the most important part of object identification and classification, in which the detection technique must identify different things from the background, whether it be a face or a hand or a man or just static objects ${ }^{5}$. In this review work, we will conclude suitable detection technique among popular techniques of detection and classification.

\section{Terminology}

We have tried to define some scenario of insects mapping with the terminology of detection technique using computer vision for better understanding. (Table-1)

\section{Table1: Basic Terminology of Insect mapped with terminology used in computer vision technique}

\section{Terminology related to Insects}

Flying insects

Insect's body structure and color combination

Flying from random position

Possible in group and count

Flying area of insects

Nocturnal flying insects

Identification of insects

Similarity in many different insect

Speed of insects

\section{Detection Techniques}

Over the years, some detection techniques have been developed. Classical methods have low impact for the discovery of object. Innovative technologies can collect data quickly and analysed in time ${ }^{6}$. In the following, popular object detection techniques are reviewed, with the objective of giving an overview of the existing technologies for object detection using computer vision.

\section{Terminology related to Computer Vision}

\section{Real-time Object}

Key points, descriptors and edge detection etc..

Key points with descriptors

Cluster identification and Segmentation

Background

Real-time object during Night time

Classification

Feature extraction, Key Points

Object motions

\section{Edge Detection}

As edge recognition is an essential step in computer vision, it is important to bring up the true edges to get the optimised outcomes from the image processing ${ }^{7}$. In this respect, Maini and Himanshu presented some pros and cons of Edge Detection Techniques (Table-2) within the conditions of object detection.

Table 2: Some pros and cons of edge detection

\begin{tabular}{|c|c|c|}
\hline Method & Pros & Cons \\
\hline $\begin{array}{l}\text { Classical(Sobel, prewitt, } \\
\text { kirsch,...) }\end{array}$ & $\begin{array}{l}\text { Simple identification of edges } \\
\text { and their orientations }\end{array}$ & $\begin{array}{l}\text { Sensitivity to noise } \\
\text { inaccurate }\end{array}$ \\
\hline $\begin{array}{l}\text { Zero crossing(Laplacian, } \\
\text { Second directional derivative) }\end{array}$ & $\begin{array}{l}\text { Identifications of edges and their. } \\
\text { orientations Having fixed characterstics } \\
\text { in all directions }\end{array}$ & $\begin{array}{l}\text { Respond to some of the existing } \\
\text { edges, sensitivity to noise }\end{array}$ \\
\hline $\begin{array}{l}\text { Laplacian of Gaussian(LoG) } \\
\text { (Marr- Hildreth) }\end{array}$ & $\begin{array}{l}\text { Finding the right places of the edges, } \\
\text { Testing large area around the pixel }\end{array}$ & $\begin{array}{l}\text { Malfunctioning at the cornes curves } \\
\text { and where the gray level intensity } \\
\text { function varies. Not finding the orientation } \\
\text { of edge because of using the Laplacian } \\
\text { filter }\end{array}$ \\
\hline $\begin{array}{l}\text { Gaussian } \\
\text { (Canny, Shen-Castan) }\end{array}$ & $\begin{array}{l}\text { error rate defined by probability, Localization } \\
\text { and response. Improving signal to consuming } \\
\text { noise ratio, Better detection specially in noise } \\
\text { conditions }\end{array}$ & $\begin{array}{l}\text { Complex Computations, Flse zero crossing, } \\
\text { Time consuming }\end{array}$ \\
\hline
\end{tabular}


After Comparison of Edge Detection technique with different conditions to all these operators, concluded that Canny's edge detection is expensive but perform well compared to Prewitt operator, Sobel operator and Robert operator ${ }^{8}$.

\section{SIFT, SURF and ORB}

Scale Invariant Feature Transform (SIFT) is a feature detector algorithm developed by David Lowe in 2004'. Speed up Robust Feature (SURF) algorithm, which is an estimation of SIFT, performs faster than SIFT without reducing the quality of the focused points ${ }^{10}$. Oriented FAST and Rotated BRIEF (ORB) as another alternative algorithm for SIFT and SURF ${ }^{11}$.

Three different image matching techniques were compared to different types of changes and disorders, such as rotation, pressure, scaling, fiasco disorder and noise. They implemented various types of changes on the original images and showed matching standards for evaluation such as the number of decimals, matching rates, and the time of execution required for each algorithm. They showed that ORB is the fastest algorithm, while SIFT execute best in most situations. For a special case when the angle of the rotation is equal to 90 degrees, the ORB and the SURF do better than the SIFT and in the pictures of the noise, ORB and SIFT show almost identical effect ${ }^{12}$.

\section{Segmentation and Recognition}

Segmentation is the process of dividing the image into its various meaningful components. Segmentation is unsupervised learning. Elements and essential measures can be understood to understand the object segmentation image. Segmentation is a distance from sensors that are based on grayscale texture, speed, depth, range, which can be used more in mobile robot training. Object segmentation has different applications, such as segmentation and diagnostic methods, identifying object size, identifying objects in the video, high-resolution video surveillance system background clutter, object dislocation and presentation in the presence of the object moved. It is very likely to improve the efficiency and accuracy of the object segmentation and the context of recognition for both images and videos $^{13}$.

\section{Convolution Neural Networks}

The Convolutional Neural Networks (CNN) are used in different applications with great performance for various task. CNN architecture had implemented the first application for applications. Recognition of handwritten digits ${ }^{14}$. There has been continuous improvement in CNN with the innovation of new layers and different computer vision techniques ${ }^{15}$. CNN holds various pre-trained model which have the capability of transfer learning so it's primary focus on the training and testing datasets at its input layer. The structure of the CNN differs in terms of techniques and layers used ${ }^{16}$.

Deep learning is about "deeper" the neural networks that pass different resolutions to provide data handshake With the simplicity of various agricultural domains, insect investigations, soil and leaf nitrogen content, plants, irrigation, medicinal uses, plant water stress, water erosion assessment, pollution identification, diagnosis of diseases against food or seed identification against crops, damage to crop damage and green home monitoring Comparative operations, along with data research areas and deep learning techniques (i.e. linear and logistic regression, Svim, Keanan, K- Unable to add the use of clustering, wavelet-based filtering, Fourier transform) analysis techniques ${ }^{17}$.

\section{Discussion}

After reviewing related work, we have found that Deep Learning has been associated with computer vision and image analysis. Deep learning based approach offers better performance comparing to Edge Detection, SIFT, SURF, ORB, Segmentation and Recognition techniques of object detection algorithm. However, reviewed paper had different datasets, preprocessing techniques and parameters; it is hard but not impossible to say performance comparison between those algorithms from papers.

\section{Conclusion}

We have looked into Convolution Neural Networks (CNN) with other popular existing techniques, in terms of performance and accuracy. Our finding indicates that CNN offers better execution and outperforms then other mainstream computer vision techniques. Our aim is that this analytical review 
would motivate more scientists to try different things with $\mathrm{CNN}$, applying it for pest detection problems including classification or prediction using computer vision and image analysis, or more generally to data analysis.

\section{References}

1. S. Narayana, "Ontology-based Advisory System for Cotton Crop Farmers," International Journal of Computer Applications. 167(7):1-7.

2. A. Rastogi, R. Arora, and S. Sharma, "Leaf disease detection and grading using computer vision technology \& fuzzy logic," in $2^{\text {nd }}$ International Conference on Signal Processing and Integrated Networks, SPIN. 2015;500-505.

3. O. S. Android, "Detection of Plant Leaf Disease Using Image Processing Approach," International Journal of Scientific and Research Publications. 2016;6(2):73-76.

4. Brian Patrick, "Invertebrates: light trapping," Department of Conservation, Government of NewZeland. 2016;1-17.

5. B. Debalina and M. Manik, "Object Identification For Computer Vision using Image Segmentation," in $2^{\text {nd }}$ International Conforence on Education Technology and Computer. 2010;170-172.

6. C. Caramuta, G. Collodel, C. Giacomini, C. Gruden, G. Longo, and P. Piccolotto, "Survey of detection techniques, mathematical models and simulation software in pedestrian dynamics," Transportation Research Procedia. 2017;25:551-567.

7. G.T.Shrivakshan, "Enhanced Edge Detection Techniques for Identification of fish through its Morphological Features," International Journal of Computer Science and Mobile Computing. 2017;6(11):48-53.

8. R. Maini and A. Himanshu, "Study and Comparison of Various Image Edge Detection Techniques," International Journal of Image Processing (IJIP). 2009;3(1):1-12.

9. D. G. Lowe, "Distinctive image features from scale-invariant keypoints," International Journal of Computer Vision. 2004;60(2): 91-110.
10. H. Bay, A. Ess, T. Tuytelaars, and L. Van Gool, "Speeded-Up Robust Features (SURF)," Computer Vision and Image Understanding. 2008;110(3):346-359.

11. E. Rublee, V. Rabaud, K. Konolige, and G. Bradski, "ORB: An efficient alternative to SIFT or SURF," in Proceedings of the IEEE International Conference on Computer Vision. 2011;2564-2571.

12. E. Karami, S. Prasad, and M. Shehata, "Image Matching Using SIFT, SURF, BRIEF and ORB: Performance Comparison for Distorted Images Image Matching Using SIFT, SURF, BRIEF and ORB : Performance Comparison for Distorted Images," in Newfoundland Electrical and Computer Engineering Conference. 2015;4.

13. A. Sharma, P. K. Singh, and P. Khurana, "Analytical Review on Object Segmentation and Recognition," in IEEE $6^{\text {th }}$ International Conference - Cloud System and Big Data Engineering (Confluence). 2016;524-530.

14. Y. LeCun, L. Bottou, Y. Bengio, and P. Haffner, "Gradient-based learning applied to document recognition," Proceedings of the IEEE. 1998;86(11):2278-2323.

15. N. Srivastava, G. Hinton, A. Krizhevsky, I. Sutskever, and R. Salakhutdinov, "Dropout: A Simple Way to Prevent Neural Networks from Overfitting," Journal of Machine Learning Research. 2014;15:1929-1958.

16. N. Sharma, V. Jain, and M. Anju, "An Analysis Of Convolutional Neural Networks For Image Classification," in Procedia Computer Science. 2018;132:377-384.

17. A. Kamilaris and F.X. Prenafeta-Boldú, "Deep learning in agriculture: A survey," Computers and Electronics in Agriculture. 2017;147: 70-90. 